\title{
Antipersistent Random Walk in a Two State Flashing Magnetic Potential
}

\author{
Pietro Tierno, ${ }^{1, *}$ Francesc Sagués, ${ }^{2}$ Tom H. Johansen, ${ }^{3,4}$ and Igor M. Sokolov ${ }^{5}$ \\ ${ }^{1}$ Estructura i Constituents de la Matèria, Universitat de Barcelona, Avenida Diagonal 647, 08028 Barcelona, Spain \\ ${ }^{2}$ Departament de Química Física, Universitat de Barcelona, Avenida Diagonal 647, 08028 Barcelona, Spain \\ ${ }^{3}$ Department of Physics, University of Oslo, P. O. Box 1048, Blindern, Norway \\ ${ }^{4}$ Centre for Advanced Study at The Norwegian Academy of Science and Letters, 0271 Oslo, Norway \\ ${ }^{5}$ Institut für Physik, Humboldt-Universität zu Berlin, Newtonstrasse 15, 12489 Berlin, Germany
}

(Received 28 February 2012; published 16 August 2012)

\begin{abstract}
We reveal the subdiffusive dynamics of paramagnetic colloids subjected to a two state flashing potential generated by periodic modulation of a magnetic bubble lattice. The particles perform a random walklike motion with antipersistent nature, showing for certain field parameters a crossover from subdiffusive to an enhanced diffusive behavior. We elucidate the stationary nature (no aging) of the subdiffusive process and stress its similarity with the random walk on a random walk model.
\end{abstract}

DOI: 10.1103/PhysRevLett.109.070601

PACS numbers: 05.40.Fb, 05.60.Cd, 82.70.Dd

Self-diffusion of colloidal particles arises due to continuous transfer of momentum by the solvent molecules [1], and it is characterized by a mean square displacement (MSD) proportional to time. In complex environments, like in crowded suspensions of hard spheres [2], granular materials [3], viscoelastic media [4], and in many biological systems [5], diffusion is often anticipated by subdiffusion, where the exponent of the power law in the MSD is less than one. Subdiffusion is usually attributed to trapping or obstruction, and understanding its origin is crucial in both fundamental [6] and applied research [7]. When dealing with individual colloids in simple fluids, the absence of interacting neighbors excludes subdiffusion a priori, which, however, can be observed by placing traps or obstacles along the particle path via chemical modification or physical actuation. In the first case, for instance, one can functionalize the particle surface and a nearby substrate with complementary strands of DNA making them "sticky" at a temperature close to the melting of the DNA [8]. On the other hand, there are many ways to manipulate colloidal particles via external fields that may be employed to confine or release the particles via remote control. In particular, magnetically patterned substrates have shown such capabilities with magnetic colloids [9], opening up the possibilities to induce anomalous kinetics in systems showing otherwise conventional diffusion.

In this context, we report on the (sub-)diffusive behavior of paramagnetic colloids moving through a flashing potential obtained via external modulation of the stray field of a magnetic bubble lattice. Depending on the applied field parameters, we observe different regimes of motion ranging from trapping to enhanced (nonthermal) diffusion. In particular, we observe robust subdiffusive motion, with MSD growing as $\sqrt{t}$ and lasting in some cases, up to three orders of magnitude in time. In the subdiffusive regime, the particles perform an antipersistent random walk with an astonishing similarity to the random walk on a random walk (RWRW) model introduced in [10], as a nontrivial example of correlated RW. Our results also demonstrate that flashing potentials, which often have been employed to ratchet molecules and colloids in the presence of nonnegligible thermal fluctuations [11], can be used to induce and conveniently control the diffusive properties of the particles.

Figure 1(a) shows a schematic of our experimental system. We use paramagnetic colloids with diameter $d=2.8 \mu \mathrm{m}$ (Dynabeads M-270) dispersed in water and deposited above the surface of a ferrite garnet film (FGF) with uniaxial anisotropy. The FGF has composition $\mathrm{Y}_{2.5} \mathrm{Bi}_{0.5} \mathrm{Fe}_{5-q} \mathrm{Ga}_{q} \mathrm{O}_{12}(q=0.51)$, a thickness of $\sim 5 \mu \mathrm{m}$, and saturation magnetization $M_{s}=1.7 \times 10^{4} \mathrm{~A} / \mathrm{m}$, and was grown by dipping liquid phase epitaxy on a (111) oriented gadolinium gallium garnet substrate. The FGF was previously modified to display a hexagonal array of magnetic "bubbles". These are cylindrical ferromagnetic domains of radius $R=4.2 \mu \mathrm{m}$, lattice constant $a=11.6 \mu \mathrm{m}$, all with the same out-of-plane magnetization and regularly arranged in a film of reverse magnetization. The size of these domains can be easily manipulated with a magnetic field applied perpendicular to the film. In particular, a field, $\mathbf{H}=H_{0} \mathbf{e}_{z}$, parallel (antiparallel) to the bubble magnetization increases (decreases) the size of the bubbles.

In contrast to Ref. [12], where a precessing field was applied to drive the particles into rotating orbits or ballistic trajectories, we here use a square-wave field, $\mathbf{H}=H_{0} \operatorname{sgn}(\sin (\omega t)) \mathbf{e}_{z}$, where $\operatorname{sgn}(x)$ is the signum function and $\omega$ denotes the frequency. The field switches between $\pm H_{0}$ and periodically changes the radii of the bubbles, flashing the potential between two states $(1 \leftrightarrow 2)$. We experimentally observe, and independently confirm through numerical calculations, Fig. 1(c), that when $H_{0}>0.1 M_{s}$, the field expands the bubbles (state 1) and the energy displays a paraboloidlike minimum within the 
(a)

$\mathrm{H}$
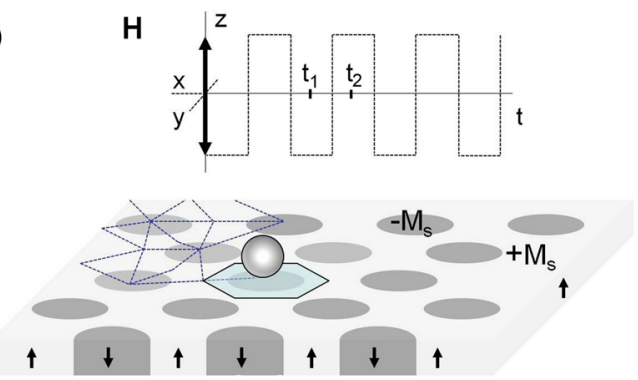

(b)

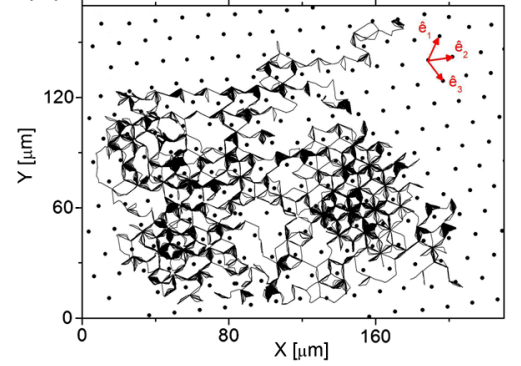

(c)

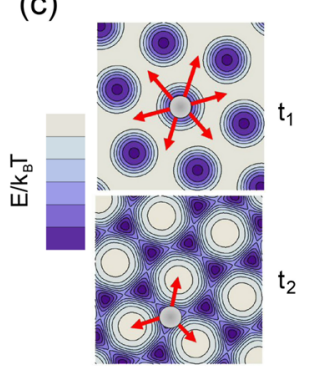

FIG. 1 (color online). (a) Scheme showing a magnetic bubble lattice with magnetization $M_{s}$ [the Wigner-Seitz cell is shaded in blue (gray)] with a paramagnetic particle on top of it subjected to a square wave magnetic field $\mathbf{H}$ normal to the film. (b) Representative trajectory of one particle $\left(\omega=12.6 \mathrm{~s}^{-1}\right.$, $H_{0}=0.14 M_{s}$ ), where the black points denote the position of the centers of the magnetic bubbles. (c) Contour plots of the normalized magnetostatic energy, $E / k_{B} T$, of one particle above the magnetic bubble lattice at two different times separated by half a period. The energy bar goes from $-10^{3} k_{B} T$ (white) to $-10^{5} k_{B} T$ [blue (gray)].

magnetic domains, while when the field shrinks the bubbles (state 2), six regions of energy minima with triangular shape appear at the vertices of the Wigner-Seitz cell around each bubble (details are given in Ref. [13]). As a consequence, during the transition $1 \rightarrow 2$, a particle can jump to 6 possible places [top of Fig. 1(c)], while in the transition $2 \rightarrow 1$, the possibilities reduce to 3 [bottom of Fig. 1(c)]. In both states, the potential preserves its spatial symmetry; i.e., no dynamical symmetry breaking occurs unlike what happens in [12,14], and after $N$ cycles the particle motion resembles a random walk on a hexagonal lattice, as shown in Fig. 1(b) (see video in [13]). The length of the walker step is given by one side of the Wigner-Seitz cell, $a / 2=5.8 \mu \mathrm{m}$, while the time between steps is controlled by the applied frequency, i.e., $t_{s}=\pi / \omega$.

We record the position of a particle at $60 \mathrm{fps}$ for $\sim 30$ min inside the observation area $\left(179 \times 231 \mu \mathrm{m}^{2}\right)$, and measure the MSD as a temporal moving average, $\left\langle\left(x\left(t^{\prime}\right)-x\left(t+t^{\prime}\right)\right)^{2}\right\rangle \sim t^{\alpha}$, where $x$ denotes the position of the particle projected onto one of the crystallographic axes of the lattice [15]. The exponent $\alpha$ of the power law distinguishes the diffusive $(\alpha=1)$ from the subdiffusive $(\alpha<1)$ dynamics.

In Fig. 2(a), we show the effect of varying the amplitude of the applied field $H_{0}$ at constant frequency $\left(\omega=6.2 \mathrm{~s}^{-1}\right)$. Small fields, $H_{0}<0.1 M_{s}$, are not able to modify the original landscape, and during the transition from $2 \rightarrow 1$, the particle can only return back to the bubble it left previously. As a consequence, the particle performs localized oscillations and the corresponding MSD saturates very fast. Increasing $H_{0}$ modifies the magnetic landscape as indicated above, and the particle is capable to leave one bubble, eventually passing to a different one during the transition $2 \rightarrow 1$. For $H_{0}=0.13 M_{s}$, the MSD shows an initial subdiffusive regime, characterized by an exponent $\alpha<1$, followed by a diffusive mode after a crossover time $\tau \sim 10 \mathrm{~s}$. The same exponent was observed at different amplitudes, $H_{0} \in[0.1,0.28] M_{s}$, while $\tau$ decreases by increasing the field strength, and for $H_{0} \geq 0.29 M_{s}$ the subdiffusion is

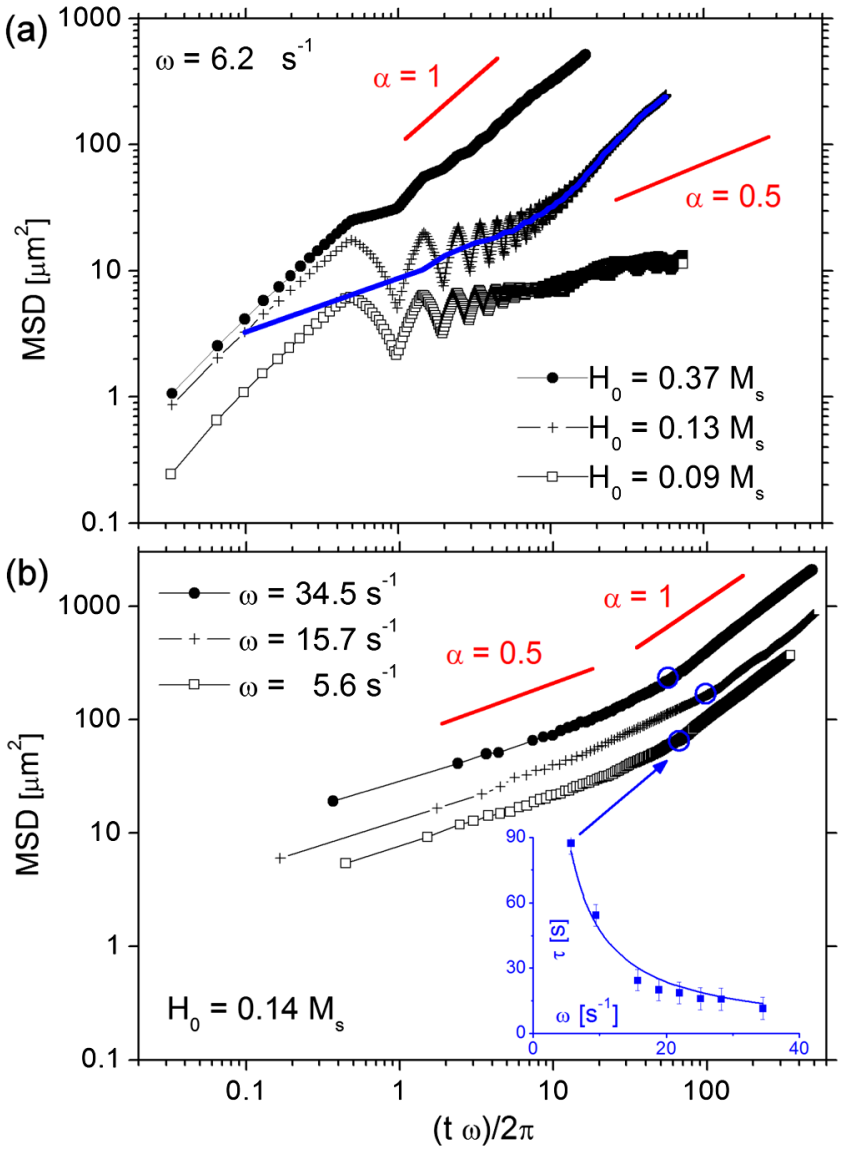

FIG. 2 (color online). (a) MSDs versus time for a paramagnetic particle under an external field with $\omega=6.2 \mathrm{~s}^{-1}$ and $H_{0}=0.09 M_{s}$ (empty squares), $H_{0}=0.13 M_{s}$ (crosses), continuous line denotes average over the oscillations, and $H_{0}=0.37 M_{s}$ (filled circles). (b) Smoothened MSDs (oscillations averaged out) versus time for an applied field with fixed $H_{0}=0.14 M_{s}$ and different frequencies, $\omega=5.6 \mathrm{~s}^{-1}$ (empty squares), $\omega=15.7 \mathrm{~s}^{-1}$ (crosses), and $\omega=34.5 \mathrm{~s}^{-1}$ (filled circles). Inset shows the crossover time $\tau$ [blue (gray)] from subdiffusive to diffusive behavior versus the frequency $\omega$ with a power law fit (continuous line). 
completely lost. In this case, the particles behave as random walkers on a hexagonal lattice from the very beginning. The diffusion coefficient can be estimated by using the expression for the random walk on isotropic lattices, $D=\frac{l^{2}}{4 t_{s}}$ where $l=a / 2$ is the distance between traps [16]. In particular, for $H_{0}=0.37 M_{s}$ we find that $D_{\exp }=$ $\lim _{t \rightarrow \infty} \operatorname{MSD}(t) / 2 t=14.6 \mu \mathrm{m}^{2} \mathrm{~s}^{-1}$ and the previous estimation gives $D=15.0 \mu \mathrm{m}^{2} \mathrm{~s}^{-1}$. We stress that the diffusion coefficient $D_{\text {exp }}$, arising from nonthermal fluctuations, exhibits a giant enhancement by two decades as compared to the thermal diffusion coefficient of a paramagnetic colloid on a glass plate $\left(0.14 \mu \mathrm{m}^{2} \mathrm{~s}^{-1}\right.$ [17]), and five decades larger than on a garnet film with no applied field $\left(\sim 10^{-4} \mu \mathrm{m}^{2} \mathrm{~s}^{-1}[18]\right)$. We note that the oscillations in the MSDs of Fig. 2(a) result from the periods of local back and forth motion of the particles, and are averaged out in Fig. 2(b).

In a second set of experiments, we measure the extension of the subdiffusive regime by keeping fixed the amplitude $\left(H_{0}=0.14 M_{s}\right)$ and varying the frequency of the field. As shown in Fig. 2(b), $\tau$ decreases with increasing frequency, and can be well fitted with a power law, $\tau \sim \omega^{-1}$ (inset). More importantly, the value of the subdiffusive exponent is quite robust, remaining constant over the whole range of frequencies, falling within the range of $\alpha \in[0.43 \ldots 0.58]$. The observed random walks display notorious features of subdiffusion pertinent to hindered motions, i.e., diffusion on a self-similar structure. We later interpret this behavior on physical grounds, but first, we will rationalize our observations in terms of an appropriate subdiffusive model. These models can be essentially divided into two classes: the models with stationary increments, and those intrinsically with nonstationary ones. Fractional Brownian motion and the random walk on a random walk model (RWRW) [10], which describes the random motion of a tagged particle on a random path, fall in the first class. Continuous time random walk (CTRW) models like the comb model [19] mimicking trapping, belong instead to the second class. In our case, the absence of aging suggests that we have to do with a model with stationary increments. This property is demonstrated in Fig. 2 of Ref. [13] as a constant ratio $\operatorname{MSD}(t) / \sqrt{t}$, which definitively rules out CTRWlike models.

To quantify the characteristics of the motion, we refer to two direct observables: the position-based probability distribution function (PDF) and the step-step autocorrelation function, defined as $C_{s}=\langle\mathbf{s}(0) \cdot \mathbf{s}(t)\rangle /\left\langle s(0)^{2}\right\rangle$ where $\mathbf{s}(\mathbf{t})$ denotes the walker step at time $t$. It is apparent from direct observations of the wandering motion of the particles (see Fig. 1 in [13]) that successive displacements of our walkers on the lattice are far from being independent. This translates into an oscillating function that evidences the antipersistent nature of the random walk; i.e., after a completed jump, a particle has an increased probability for returning to its previous location compared to going elsewhere.
The step-step correlation function (inset of Fig. 3) resembles to a large extent that of the RWRW model. This is stressed by the similarity of the decay $(1 / \sqrt{t})$ of the envelopes of $C_{s}$ in both situations as displayed in Fig. 3, where the experimentally obtained $\left|C_{s}(t)\right|$ is compared to the simulated one for RWRW. The two correlation functions show very similar behavior until the one for the experimental system starts to be dominated by noise.

Let us now turn to the form of the PDF for the time domain when the motion is not yet dominated by noise. Although the PDF of the process is not uniquely determined by its correlation function, again the corresponding PDFs in our experimental system and in the RWRW model are strikingly similar. In the RWRW model, this PDF is non-Gaussian and given by the integral expression [20],

$$
p(x, b)=\frac{1}{\pi} \sqrt{\frac{1}{b}} \int_{0}^{\infty} \frac{1}{\sqrt{s}} \exp \left(-\frac{x^{2}}{2 s}-\frac{s^{2}}{2 b}\right) d s
$$

with the parameter $b$ given by the the dispersion of the observed distribution via $\sigma=\sqrt{\frac{2}{\pi b}} \int_{0}^{\infty} s \exp \left(-\frac{s^{2}}{2 b}\right) d s=$ $\sqrt{b / 2 \pi}$. In particular, we measure the PDF of the variance $\delta x_{N}=x_{i}-x_{i+N}$, with $N$ being the number of elapsed frames. Figure 4 shows the normalized PDFs for a particle under a field with $H_{0}=0.14 M_{s}$ and $\omega=25.1 \mathrm{~s}^{-1}$, corresponding to a crossover in the MSD occurring after c.a. 900 frames. For $N=10$ the PDF is clearly non-Gaussian, with a central peak decreasing as $N$ increases. It shows an excellent agreement with the corresponding PDF of the RWRW. On the other hand, in the long time diffusive regime, when the correlation function is dominated by noise (which is the case, e.g., for $N=1000$ ), the PDF can be well fitted to a Gaussian by using the same dispersion $\sigma$.

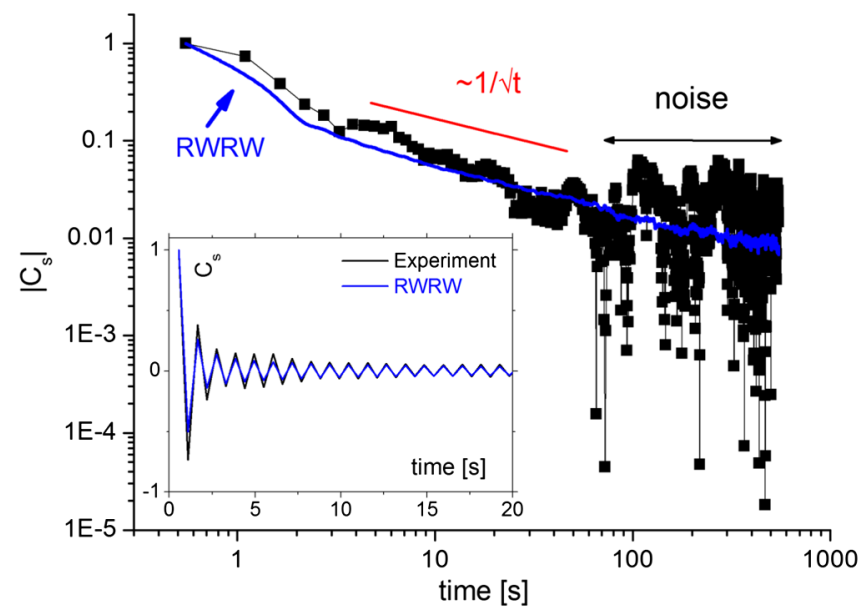

FIG. 3 (color online). Absolute value of the normalized stepstep correlation function $\left|C_{s}\right|$ versus time for a paramagnetic colloid subjected to an external field with $\omega=25.1 \mathrm{~s}^{-1}$ and $H_{0}=0.14 M_{s}$. Gray [blue (gray)] line denotes the $\left|C_{s}\right|$ calculated for the RWRW model. Inset shows the oscillations of $C_{s}$. 


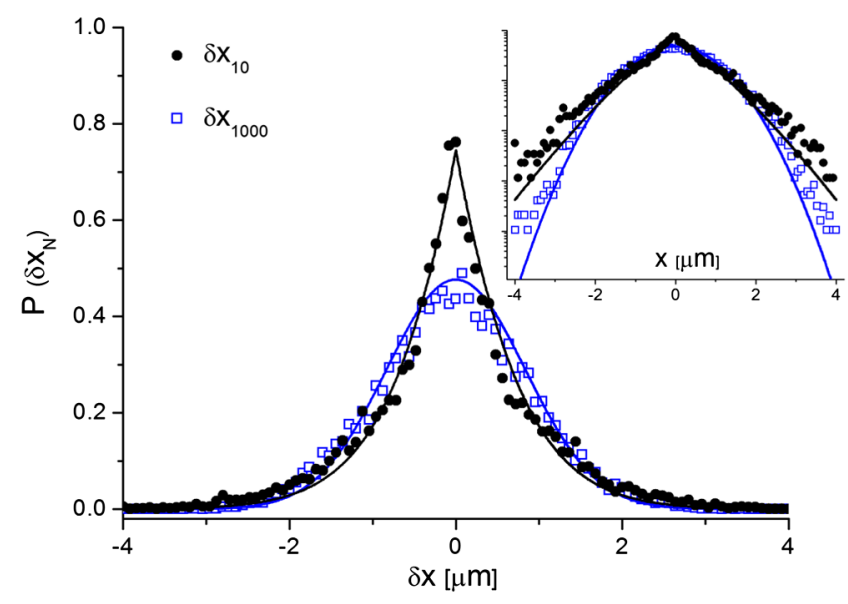

FIG. 4 (color online). Normalized PDFs of the variance $\delta x_{N}=$ $x_{i}-x_{i+N}$, with $N$ the number of frames. The continuous lines are fits using Eq. (1) in the text for $N=10$ (black) and a Gaussian distribution for $N=1000$ [blue (gray)]. Inset shows the same graph in semilogarithmic scale to visualize the PDFs tails.

To justify the origin of subdiffusion, let us consider the response of the magnetic bubbles to the applied field. The magnetic bubbles exhibit a certain degree of randomness in their shape during expansion or contraction due to the presence of pinning sites and other inhomogeneities which exert an effective frictional force against their motion [21]. These deformations are not reproducible upon reapplication of the field and, as a consequence, they translate into a dynamic disorder. This dynamic disorder, which was considered in [22] as bubble "polydispersity", creates lattice neighbourhoods where the spatial symmetry of the energy minima around the bubbles is temporary lost. As a consequence of this disorder, one particle may occasionally encounter a region in which its dispersive motion is hindered while continuously moving back and forth between neighbouring bubbles. The dependence of the subdiffusive regime with the frequency can be explained by considering the behavior of a system under periodic forcing with low disorder. When the forcing is too fast, the particle feels an average potential coming from the vibrations of the bubbles and its trajectory remains unperturbed with respect to the situation in absence of disorder. In contrast, at low frequencies, the particle is capable to explore the effective potential, and the effect of this disorder appears.

To conclude, we found enhanced diffusion and subdiffusion in a system of paramagnetic colloids subjected to a flashing magnetic potential and demonstrate the correlated antipersistent nature of the particle random walk similar to the RWRW. Although subdiffusion in percolationlike structures has been observed in soft matter system, like reptation in polymers and biopolymers, this study is the first experimental demonstration of this type of subdiffusion in periodic potentials. Thus, the proposed method provides a new model system to study anomalous transport phenomena arising from dynamic disorder, a situation encountered in many other physical systems ranging from charge transport in random potentials [23], membrane rafts [24] to proteins in cytoplasmic barriers [25].

P. T. was supported by the "Ramon y Cajal" program (RYC-2011-07605). P. T. and F. S. acknowledge support by MEC (FIS201021924C02, FIS201113771E) and DURSI (2009SGR1055). T.H.J. by The Research Council of Norway.

*ptierno@ub.edu

[1] A. Einstein, Ann. Phys. (Leipzig) 322, 549 (1905); 324, 289 (1906).

[2] E. R. Weeks and D. A. Weitz, Phys. Rev. Lett. 89, 095704 (2002); Chem. Phys. 284, 361 (2002).

[3] G. Marty and O. Dauchot, Phys. Rev. Lett. 94, 015701 (2005); P. M. Reis, R. A. Ingale, and M. D. Shattuck, Phys. Rev. Lett. 98, 188301 (2007).

[4] F. Amblard, A.C Maggs, B. Yurke, A. N. Pargellis, and S. Leibler, Phys. Rev. Lett. 77, 4470 (1996); S. C. Weber, A. J. Spakowitz, and J. A. Theriot, Phys. Rev. Lett. 104, 238102 (2010).

[5] G. Seisenberger, M.U. Ried, T. Endreß, H. Büning, M. Hallek, and C. Bräuchle, Science 294, 1929 (2001); I. M. Tolić-Nørrelykke, E. L. Munteanu, G. Thon, L. Oddershede, and K. Berg-Sorensen, Phys. Rev. Lett. 93, 078102 (2004); I. Golding and E. C. Cox, Phys. Rev. Lett. 96, 098102 (2006); J. Szymanski and M. Weiss, Phys. Rev. Lett. 103, 038102 (2009); T. Akimoto, E. Yamamoto, K. Yasuoka, Y. Hirano, and M. Yasui, Phys. Rev. Lett. 107, 178103 (2011).

[6] J.-P. Bouchaud and A. Georges, Phys. Rep. 195, 127 (1990); R. Metzler and J. Klafter, Phys. Rep. 339, 1 (2000); P. Hänggi and F. Marchesoni, Rev. Mod. Phys. 81, 387 (2009); Y. Meroz, I. M. Sokolov, and J. Klafter, Phys. Rev. E 81, 010101 (2010); P. Siegle, I. Goychuk, and P. Hänggi, Phys. Rev. Lett. 105, 100602 (2010).

[7] B. Li and J. Wang, Phys. Rev. Lett. 91, 044301 (2003); I. Y. Wong, M. L. Gardel, D. R. Reichman, E. R. Weeks, M. T. Valentine, A. R. Bausch, and D. A. Weitz, Phys. Rev. Lett. 92, 178101 (2004); G. Marty and O. Dauchot, Phys. Rev. Lett. 94, 015701 (2005); K. Henzler, S. Rosenfeldt, A. Wittemann, L. Harnau, S. Finet, T. Narayanan, and M. Ballauff, Phys. Rev. Lett. 100, 158301 (2008).

[8] Q. Xu, L. Feng, R. Sha, N. C. Seeman, and P. M. Chaikin, Phys. Rev. Lett. 106, 228102 (2011).

[9] L.E. Helseth, T. M. Fischer, and T. H. Johansen, Phys. Rev. Lett. 91, 208302 (2003); P. Tierno, F. Sagués, T. H. Johansen, and T. M. Fischer, Phys. Chem. Chem. Phys. 11, 9615 (2009); M. Donolato et al., Adv. Mater. 22, 2706 (2010).

[10] K. W. Kehr and R. Kutner, Physica (Amsterdam) 110A, 535 (1982).

[11] R. D. Astumian and M. Bier, Phys. Rev. Lett. 72, 1766 (1994); L. P. Faucheux, L. S. Bourdieu, P. D. Kaplan, and A. J. Libchaber, Phys. Rev. Lett. 74, 1504 (1995); P. Reimann, Phys. Rep. 290, 149 (1997); F. J. Cao, L. Dinis, and J.M.R. Parrondo, Phys. Rev. Lett. 93, 040603 (2004); T. Harada and K. Yoshikawa, Phys. Rev. E 69, 031113 (2004); A. Libál, C. Reichhardt, B. Janko, 
and C. J. Olson Reichhardet, Phys. Rev. Lett. 96, 188301 (2006); B. J. Lopez, N. J. Kuwada, E. M. Craig, B. R. Long, and H. Linke, Phys. Rev. Lett. 101, 220601 (2008).

[12] P. Tierno, T. H. Johansen, and T. M. Fischer, Phys. Rev. Lett. 99, 038303 (2007).

[13] See Supplemental Material at http://link.aps.org/ supplemental/10.1103/PhysRevLett.109.070601 for a detailed explanation of the numerical method used for Fig. 1(c), the absence of aging in the MSD, and one video showing the particle dynamics in the flashing potential.

[14] C. Jungnickel, Z. Khattari, T. H. Johansen, and Th. M. Fischer, New J. Phys. 13, 043014 (2011).

[15] To confirm the system isotropy, we measure the MSD along two lattice directions $\mathbf{e}_{1} \equiv\left(\frac{1}{2} a, \frac{\sqrt{3}}{2} a\right), \mathbf{e}_{2} \equiv(a, 0)$, and observe the same results.

[16] J. Machta and R. Zwanzig, Phys. Rev. Lett. 50, 1959 (1983); R. Klages and C. Dellago, J. Stat. Phys. 101, 145 (2000).
[17] P. Tierno, R. Muruganathan, and T. M. Fischer, Phys. Rev. Lett. 98, 028301 (2007).

[18] P. Tierno, P. Reimann, T.H. Johansen, and F. Sagues, Phys. Rev. Lett. 105, 230602 (2010).

[19] G. H. Weiss and S. Havlin, Physica (Amsterdam) 134A, 474 (1986)

[20] Y. Meroz, I. M. Sokolov, and J. Klafter, Phys. Rev. Lett. 107, 260601 (2011).

[21] V. G. Bar'yakhtar, V. V. Gann, Yu. I. Gorobets, G. A. Smolenski, and B. N. Filippov, Sov. Phys. Usp. 20, 298 (1977).

[22] A. Soba, P. Tierno, T. M. Fischer, and F. Sagues, Phys. Rev. E 77, 060401 (2008).

[23] R. Richert, L. Pautmeier, and H. Bässler, Phys. Rev. Lett. 63, 547 (1989).

[24] J. Ehrig, E. P. Petrov, and P. Schwille, Biophys. J. 100, 80 (2011).

[25] M. Edidin, S. C. Kuo, and M. P. Sheetz, Science 254, 1379 (1991). 